\title{
Status of and Trends in Nuclear Medicine in the United States
}

\author{
Dominique Delbeke ${ }^{1}$ and George M. Segall ${ }^{2,3}$ \\ ${ }^{I}$ Vanderbilt University Medical Center, Nashville, Tennessee; ${ }^{2}$ Palo Alto VA Health Care System, Palo Alto, California; and ${ }^{3}$ Stanford \\ University, Stanford, California
}

Nuclear medicine in the United States has grown because of advances in technology, including hybrid imaging, the introduction of new radiopharmaceuticals for diagnosis and therapy, and the development of molecular imaging based on the tracer principle, which is not based on radioisotopes. Continued growth of the field will require cost-effectiveness data and evidence that nuclear medicine procedures affect patients' outcomes. Nuclear medicine physicians and radiologists will need more training in anatomic and molecular imaging. New educational models are being developed to ensure that future physicians will be adequately prepared.

Key Words: nuclear medicine; statistics; status; trends

J Nucl Med 2011; 52:24S-28S

DOI: 10.2967/jnumed.110.085688

$\mathbf{N}$ uclear medicine has a history of decades of strong growth, particularly in nuclear cardiology and PET/CT. In the United States, the number of nuclear medicine procedures has grown from approximately 14 million in 1999 to almost 20 million in 2005 (1). After 2005, however, it dropped to about 17 million. Most nuclear medicine procedures are performed in hospital-based settings, but the number performed in nonhospital settings has grown over time to one third of all nuclear medicine procedures in 2008 .

In the United States, the growth of nuclear medicine procedures is due primarily to nuclear cardiology, which has grown from about 7 million procedures in 1999 to about 11 million in 2005 (1). Nuclear cardiology represents more than $50 \%$ of the nuclear medicine procedures done in the United States but represents only 14\% of those done in Europe. A study performed in 2007 investigated the worldwide use of nuclear cardiology (2). The study findings indicated that nuclear cardiology procedures were used most extensively in the United States, with 1,000 or more procedures performed per 100,000 people (Fig. 1).

Bone scintigraphy is the next most common nuclear medicine procedure performed in the United States, but it

Received Aug. 25, 2011; revision accepted Nov. 2, 2011.

For correspondence or reprints contact: George M. Segall, Nuclear Medicine Service 115, Palo Alto VA Health Care System, 3801 Miranda Ave., Palo Alto, CA 94304.

E-mail: george.segall@va.gov

COPYRIGHT @ 2011 by the Society of Nuclear Medicine, Inc. represents only $17 \%$ of procedures (1). Bone scintigraphy represented 36\% of procedures performed in Europe in 2008.

The volume of PET procedures in the United States is approximately 1.5 million per year (3). Oncology accounts for more than $90 \%$ of the PET and PET/CT procedures performed, whereas cardiology and neurology account for about $4 \%$ each (3). According to the National Oncology PET Registry, about $84 \%$ of the slightly more than 1,600 PET facilities in the United States have PET/CT systems (4). The emergence of molecular imaging with new radiopharmaceuticals and new technologies is likely to result in continued growth in the coming decades.

Efforts to control spiraling health care costs have resulted in decreased reimbursement for medical imaging and the need to provide evidence that patients' outcomes are improved by diagnostic tests and therapies. Radiology benefit managers have become gatekeepers for insurance plans, but without evidence to ensure the appropriate use of medical imaging, coverage decisions are frequently based on cost. Downward pressure on nuclear medicine is also being exerted by heightened concern about radiation exposure and the recent worldwide shortage of ${ }^{99} \mathrm{Mo}$.

The cost of advanced imaging procedures has grown disproportionately compared with the overall cost of health care. The goals of health care reform are to provide health care to more people and to control rising costs. Methods to achieve these goals include shifting expenditures from specialized care to primary care and preventive medicine and replacing a fee-for-service system with a payment system based on quality of care. Laboratory accreditation and physicians' adherence to evidenced-based practice guidelines and appropriateness criteria will be increasingly important conditions for payment.

Nuclear medicine studies may change medical management (4). Nuclear medicine studies have been shown to be cost-effective $(5,6)$. For example, the Economics of Noninvasive Diagnosis study, a prospective study of 11,372 consecutive patients who had stable angina and were referred for stress myocardial perfusion tomography or cardiac catheterization, demonstrated that costs of care were higher for direct cardiac catheterization in all clinical risk subsets (range, \$2,878-\$4,579) than for stress myocardial perfusion imaging plus selective catheterization (range, $\$ 2,387-\$ 3,010)(P<0.0001)(7)$. Coronary revascularization rates were higher for patients who had low, intermediate, 


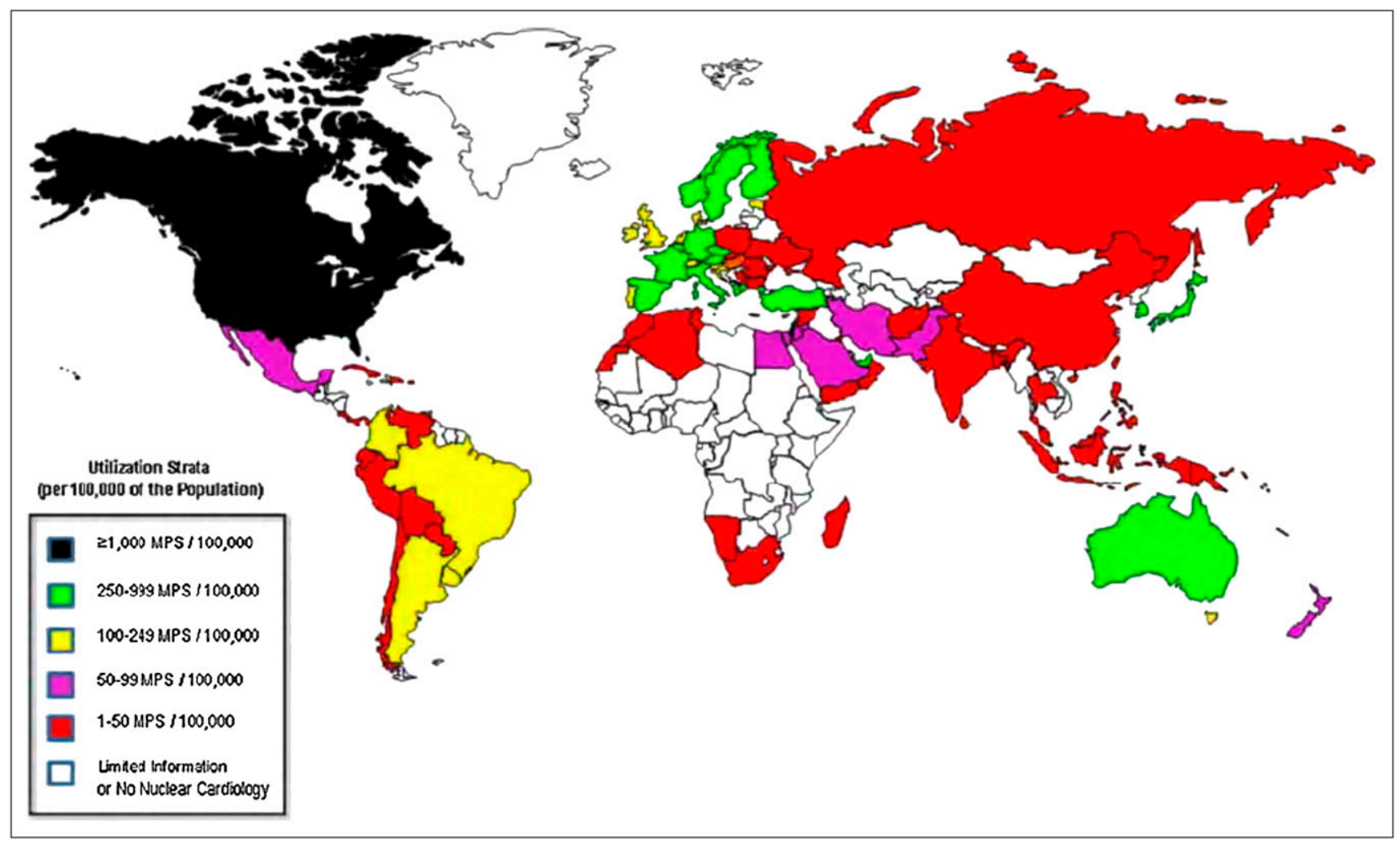

FIGURE 1. Estimates of worldwide use (per 100,000 people) of nuclear cardiology procedures. Data were based on 2008 survey of number of annual nuclear cardiology procedures relative to 2007 population statistics. MPS = myocardial perfusion scintigraphy. (Reprinted with permission of (2).)

and high risk and who received direct cardiac catheterization than for patients who received initial stress myocardial perfusion imaging $(13 \%-50 \%)(P<0.0001)$; cardiac death or myocardial infarction rates were similar $(P>0.20)(7)$.

In a recent review ( 6 ) of the economic evaluation of PET, excluding health technology assessments, 14 publications met the inclusion criteria for demonstrating cost-effectiveness; all of them were model based and included diagnosis of a solitary pulmonary nodule $(n=1)$, staging of recurrent ovarian cancer $(n=1)$, staging of liver metastases from colorectal cancer $(n=1)$, staging of pulmonary metastases from malignant melanoma $(n=1)$, staging of recurrent nasopharyngeal carcinoma $(n=1)$, staging of head and neck cancer $(n=1)$, staging of breast cancer $(n=1)$, follow-up of non-small cell lung cancer $(n=1)$, and staging of non-small cell lung cancer $(n=6)$.

Comparative effectiveness studies and well-designed clinical trials are necessary to provide a sound scientific foundation for clinical acceptance of advanced imaging procedures, such as PET/CT and SPECT/CT, especially with new tracers.

Economic pressures are decreasing revenues for professional medical organizations, requiring a reexamination of priorities to balance expenses with revenues. Health care professionals have less time and fewer financial resources to support and participate in the activities of professional organizations.
The medical specialty of nuclear medicine faces significant challenges because of the intersection with radiology, which has accelerated since the introduction of hybrid imaging, and the evolution of molecular imaging. Professional radiology organizations, such as The American Board of Radiology (ABR), American College of Radiology (ACR), and Radiological Society of North America, are playing increasing roles in setting professional standards and providing education for nuclear medicine professionals.

Economic pressures have increased competition among professional organizations. Radiology organizations enjoy a significant advantage over nuclear medicine organizations because of their large size, which provides them with more funds, more people, and more infrastructure. Radiologists also significantly outnumber nuclear medicine physicians. In May 2011, 1,361 candidates took the diagnostic radiology oral examination given by ABR (8); however, only 87 candidates took the examination given by the American Board of Nuclear Medicine (ABNM) in October 2011.

\section{TRAINING IN NUCLEAR MEDICINE}

There are multiple nuclear medicine training pathways in the United States; they have been summarized in the report of the ACR-Society of Nuclear Medicine (SNM) Task Force on Nuclear Medicine Training $(9,10)$. These pathways can lead to certification by 2 different member boards 
of the American Board of Medical Specialties (ABMS): $\mathrm{ABNM}$ and $\mathrm{ABR}$. ABNM considers nuclear medicine to be a primary medical specialty, which is recognized by the ABMS. ABR considers nuclear radiology to be a primary subspecialty of radiology; this consideration has caused confusion and different standards for education and practice for nuclear medicine and nuclear radiology.

To be eligible for ABNM certification, applicants must receive training in 1 of the 54 nuclear medicine resident training programs accredited by the Accreditation Council for Graduate Medical Education (ACGME) (11). There are currently 158 on-duty residents (12). The length of required nuclear medicine training depends on prior training: $3 \mathrm{y}$ of nuclear medicine after 1 clinical year, $2 \mathrm{y}$ of nuclear medicine if a physician is eligible for another specialty board certification, or $1 \mathrm{y}$ of nuclear medicine if a physician is eligible for $A B R$ certification. A fourth training pathway was approved by ABNM in 2010. Residents enrolled in a radiology resident training program are eligible for ABNM certification after completing 16 mo of training in an ACGME-accredited nuclear medicine program during their $4 \mathrm{y}$ of radiology training. Each nuclear medicine program graduates an average of 2 residents per year, for a total of about 100 residents per year. The number of physicians taking the examination for ABNM certification has been stable for the past $10 \mathrm{y}$, averaging about 90-100 annually. This number includes about $20 \%$ with a certificate from $\mathrm{ABR}$ and $5 \%$ with a certificate from the American Board of Internal Medicine. A survey of nuclear medicine program directors was conducted in 2009 by the ACR-SNM Task Force on Nuclear Medicine Training, and 22 responses were received $(9,10)$. The survey showed that about $65 \%$ of nuclear medicine residents complete a residency in diagnostic radiology either before $(30 \%)$ or after $(35 \%)$ their residency in nuclear medicine.

The ACGME program requirements for nuclear medicine specify an amount of training for the oral administration of radioiodine for therapy that exceeds the amount of training in diagnostic radiology and includes training for the parenteral administration of radiopharmaceuticals for therapy that is not included in training in diagnostic radiology. The same nuclear medicine training standards are recommended in the conjoint statement of the SNM, American College of Nuclear Medicine, and ABNM on credentialing and delineation of privileges for therapeutic procedures using radiopharmaceuticals (13). Effective July 1, 2011, nuclear medicine residents must also have a minimum of 6 mo of CT experience, including a minimum of 4 mo in a diagnostic radiology CT service (14).

To be eligible for subspecialty certification in nuclear radiology by $\mathrm{ABR}$, physicians must have $\mathrm{ABR}$ certification in diagnostic radiology and an additional year of fellowship training in nuclear radiology in 1 of the 19 ACGMEaccredited nuclear radiology resident training programs (15). There are currently 15 on-duty residents (12). This training pathway includes a total of 16 mo in nuclear radi- ology: 4 mo during radiology residency plus 12 mo during fellowship training. In 2011, ABR created a second pathway for subspecialty certification in nuclear radiology. This pathway consists of 16 mo of training in nuclear radiology or nuclear medicine during $4 \mathrm{y}$ of radiology residency; 10 mo of this training must be consecutive.

A significant difference between nuclear medicine and nuclear radiology is the amount of training required for therapy with radiopharmaceuticals. Nuclear medicine training requires experience with 10 patients receiving low-dose $(\leq 1,221 \mathrm{MBq}){ }^{131} \mathrm{I}$ therapy, 5 patients receiving high-dose $(>1,221 \mathrm{MBq}){ }^{131} \mathrm{I}$ therapy, and 3 patients receiving parenteral therapy. Nuclear radiology training does not specify the amount of training required for therapy, and ABR only requires physicians to have training experience with 3 patients receiving low-dose ${ }^{131}$ I therapy and 3 patients receiving high-dose ${ }^{131}$ I therapy before taking the certification examination in diagnostic radiology. Physicians with a diagnostic radiology certificate or a subspecialty certificate in nuclear radiology are not qualified to administer parenteral therapy, according to regulations of the Nuclear Regulatory Commission.

To be eligible for ABR certification in diagnostic radiology, physicians must complete a radiology residency with 4 mo of nuclear medicine training in 1 of the 187 ACGMEaccredited radiology residency programs (16). ABR considers physicians who are certified in diagnostic radiology to be qualified to practice the full scope of nuclear radiology. Subspecialty certification in nuclear radiology does not provide additional qualifications in radionuclide therapy.

There are currently 4,604 on-duty radiology residents (12) — about 1,000 per year of training — who are eligible to take the examination given by ABR for certification in diagnostic radiology. In response to ABR testing changes set to take place in 2013, a resident education committee was formed at the University of Virginia in 2010 to evaluate the radiology training required during the first 36 mo to prepare for the core examination (17). This committee compared the number of weeks that radiology residents spent on different rotations during $4 \mathrm{y}$ in the pre-2010 curriculum and the new (2010) curriculum (Fig. 2). In the diagnostic radiology residency program, the time spent in nuclear medicine before 2010 was $16 \mathrm{wk}$ (range, 12-16 wk); the goal in 2010 was 16 wk. The time spent in body CT before 2010 was $8 \mathrm{wk}$ (range, $8-16 \mathrm{wk}$ ); the goal in 2010 was $10 \mathrm{wk}$ (range, 6-10 wk).

\section{NUCLEAR MEDICINE AND RADIOLOGY IN CLINICAL PRACTICE}

Because of the various training pathway and specialty board certifications available in the United States, nuclear medicine is practiced by general radiologists, nuclear radiologists, and nuclear medicine physicians. There are approximately 10 times more practicing radiologists than nuclear medicine physicians. Cardiologists account for a significant percentage of nuclear cardiology practice. 
Table 2. Comparison of the number of weeks our residents spend on services in both the current and the new 2010 curricula over 4 years

\begin{tabular}{|c|c|c|}
\hline Rotation & Current & 2010 \\
\hline Chest plain film & $16(8-20)$ & $12(12)$ \\
\hline Fluoroscopy (gastrointestinal) & $12(8-18)$ & $12(12)$ \\
\hline Body CT & $8(8-16)$ & $10(6-10)$ \\
\hline Outpatient imaging center & $4(2-8)$ & $4(4-6)$ \\
\hline Ultrasound & $8(4-14)$ & $6(6)$ \\
\hline Emergency radiology & $8(4-18)$ & $6(4-6)$ \\
\hline Body MR & $4(4-8)$ & $6(6)$ \\
\hline Genitourinary (fluoroscopy and CT) & $12(2-14)$ & $6(6)$ \\
\hline Pediatric radiology & $8(8-16)$ & $8(8)$ \\
\hline Nuclear medicine & $16(12-16)^{\dagger}$ & $16(16)$ \\
\hline $\begin{array}{l}\text { Noninterventional cardiovascular } \\
\text { imaging }\end{array}$ & $4(0-4)$ & $6(6)$ \\
\hline Cardiac (nuclear medicine and CT/MR) & $4(2-6)$ & $4(4)$ \\
\hline Cross-cover (general) & $8(0-11)$ & $10(4-10)$ \\
\hline Body procedures & $4(2-8)$ & $4(4)$ \\
\hline Neuroradiology & $24(20-30)$ & $20(20)$ \\
\hline Musculoskeletal radiology & $16(8-22)$ & $12(12)$ \\
\hline Breast imaging ${ }^{*}$ & $12(12-14)$ & $12(12)$ \\
\hline Interventional radiology & $12(4-60)^{\ddagger}$ & $12(12)$ \\
\hline Night float & $12(10-14)$ & $18(12-18)$ \\
\hline Elective time & $8(2-8)$ & \\
\hline Focus time & & $16(16-32)^{\ddagger}$ \\
\hline Armed Forces Institute of Pathology & $4(4-6)$ & $4(4)$ \\
\hline Research time & $4(2-30)^{\ddagger}$ & $4(4)$ \\
\hline Total & 208 & 208 \\
\hline \multicolumn{3}{|c|}{$\begin{array}{l}\text { Data are expressed as mode (range). } \\
\text { "Length mandated by ACGME. } \\
\text { tRange includes < } 16 \text { weeks because the rotation includes nuclear medicine } \\
\text { experience and allows our residents to meet ACGME mandates. } \\
\text { FIncludes our specialty-track residents; interventional radiology and research time } \\
\text { under the current curriculum shift to focus time in the } 2010 \text { definition. }\end{array}$} \\
\hline
\end{tabular}

FIGURE 2. Comparison of core curricula in diagnostic radiology implemented at University of Virginia in 2010 and earlier. (Reprinted with permission of (17).)

There were 6,800 diplomates of the Certification Board of Nuclear Cardiology as of February 15, 2010, although not all diplomates are cardiologists (18). Endocrinologists and other specialists account for a small percentage of nuclear medicine practice.

In 2010, the ACR Web site listed approximately 34,000 members (100 radiologists per million inhabitants), 150 committees, and 1,500 volunteers (19). The Association of University Radiologists Web site listed 3,000 academic radiologists (20), and the Society of Chairs of Academic Radiology listed 165 chairs of academic radiology departments (21). The majority of radiologists $(49 \%-$ $69 \%$ ) are in private practices or radiology-only groups, $16 \%-18 \%$ are in private practices or multispecialty groups, $15 \%-20 \%$ are in academic practices, and $2 \%-3 \%$ are in government practices. Most radiologists $(75 \%)$ have subspecialty training but spend $10 \%-25 \%$ of their time practicing general radiology; $25 \%$ of radiologists practice general radiology only. The 4 largest radiology subspecialties are cross-sectional or abdominal imaging, interventional or vascular radiology, breast imaging or women's imaging, and neuroradiology. The average radiologist interprets 15,000 studies per year-approximately $20 \%$ CT and
5\% MRI. Between 1990 and 2007, the percentages of radiologists in large practices (more than 15 radiologists) and radiologists in multispecialty practices increased (22).

The SNM has approximately 17,000 members, including approximately 4,500 physicians and scientists (10 nuclear medicine physicians per million inhabitants) and 10,000 technologists, the remainder being trainees and industry members (23). International members represent $11 \%$ of the total. The SNM has 24 committees and approximately 300 volunteers. The 2009 survey of nuclear medicine program directors, with 22 responses, indicated that $40 \%$ of nuclear medicine physicians are in private practices $(9,10)$. The SNM surveyed its membership in 2010 and received 4,063 responses $(30 \%$ physicians and scientists and $57 \%$ technologists). The survey showed that $30 \%$ of nuclear medicine physicians work in non-university-affiliated hospitals or medical centers, $27 \%$ work in academic institutions, $14 \%$ work in free-standing imaging facilities, $2 \%$ work in molecular imaging laboratories, and $1 \%$ work in government laboratories.

Because most radiologists with subspecialty training spend $25 \%$ of their time practicing general radiology and are on call for general radiology, imaging physicians with training in nuclear medicine are expected to do the same, especially with increasing economic pressures; however, nuclear medicine physicians lack the required education and training in radiology. A survey of radiology chairs and nuclear medicine program directors performed in 2009 by the ACR-SNM Task Force on Nuclear Medicine Training confirmed this belief $(9,10)$ on the basis of 108 of 508 responses ( $31 \%$ of radiology chairs and $56 \%$ of nuclear medicine program directors). Although the certification of physicians interpreting nuclear medicine was about equally distributed among physicians certified by ABR only, ABNM only, and ABR plus ABNM, the employer's preference for most cases was certification by ABR plus ABNM (45\% of the respondents) or certification by ABR only ( $27 \%$ of the respondents). An important consideration for $66 \%$ of the respondents was the ability to provide coverage for general radiology and be on call, whereas advanced training in nuclear medicine and molecular imaging was important for only $22 \%$ of the respondents. A total of $44 \%$ of the respondents would hire only radiologists, whereas $56 \%$ would hire both radiologists and nuclear medicine physicians. Despite the negative impact of the economy on employment, a survey of ABNM diplomates in 2010 indicated that $82 \%$ were employed within $1 \mathrm{y}$ of ABNM certification, although only $51 \%$ were employed primarily in nuclear medicine (24). The survey was limited by undersampling, as there were only 49 responses.

\section{CONTINUING MEDICAL EDUCATION AND MAINTENANCE OF CERTIFICATION}

Evidence-based guidelines and appropriateness criteria for imaging, especially imaging with advanced or new technologies, will become increasingly important. Improvement in patients' outcomes will need to be demonstrated when imaging is included in the management algorithm. 
Recommendations for qualifications of personnel, including physicians and technologists, are usually included in guidelines or credentialing statements and are used by laboratory accreditation organizations and payers for credentialing of physicians. Board certification and continuing medical education have been mandatory for a long time for licensure maintenance.

In the year 2000, the ABMS adopted the concept of maintenance of certification (MOC), which has 4 components: professional standing, cognitive expertise, lifelong learning and self-assessment, and practice performance. MOC programs focus on 6 general competencies integral to the quality of care: patient care, medical knowledge, practice-based learning and improvement, interpersonal and communication skills, professionalism, and system-based practices. Nearly $90 \%$ of the licensed physicians in the United States are certified by at least 1 ABMS member board. Therefore, most physicians are involved in some aspects of MOC. ABNM certificates are limited to $10 \mathrm{y}$, and the first certificate expired in 2002. Participation in MOC is mandatory for physicians with time-limited certificates. All ABNM diplomates will need to pass a recertification examination by 2017. Eligibility for the recertification examination requires continuous participation in MOC. Recommendations for credentialing of physicians will increasingly require participation in MOC instead of continuing medical education only.

\section{CONCLUSION}

Nuclear medicine in the United States has grown because of advances in technology, including hybrid imaging, the introduction of new radiopharmaceuticals for diagnosis and therapy, and the development of molecular imaging based on the tracer principle, which does not use radioisotopes. Continued growth of the field will require cost-effectiveness data and evidence that nuclear medicine procedures affect patients' outcomes. Nuclear medicine physicians and radiologists will need more training in anatomic and molecular imaging. New educational models are being developed to ensure that future physicians will be adequately prepared.

\section{ACKNOWLEDGMENT}

No potential conflict of interest relevant to this article was reported.

\section{REFERENCES}

1. IMV Medical Information Division, Inc. 2008 Nuclear medicine market summary report. Available at: http://www.marketresearch.com/IMV-MedicalInformation-Division-Inc-v229/. Accessed November 15, 2011.
2. Vitola JV, Shaw LJ, Allam AH, et al. Assessing the need for nuclear cardiology and other advanced cardiac imaging modalities in the developing world. $\mathrm{J} \mathrm{Nucl}$ Cardiol. 2009; 16:956-961.

3. IMV Medical Information Division, Inc. 2008 PET market summary report. Available at: http://www.marketresearch.com/IMV-Medical-Information-DivisionInc-v229/. Accessed November 15, 2011.

4. Hillner BE, Siegel BA, Liu D, et al. Impact of positron emission tomography/ computed tomography and positron emission tomography (PET) alone on expected management of patients with cancer: initial results from the National Oncology PET Registry. J Clin Oncol. 2008;26:2155-2161.

5. Des Prez RD, Shaw LJ, Gillespie RL, et al. Cost-effectiveness of myocardial perfusion imaging: a summary of the currently available literature. $\mathrm{J} \mathrm{Nucl} \mathrm{Cardiol}$. 2005;12:750-759.

6. Langer A. A systematic review of PET and PET/CT in oncology: a way to personalize cancer treatment in a cost-effective manner? BMC Health Serv Res. 2010;10:283.

7. Shaw LJ, Hachamovitch R, Berman DS, et al. The economic consequences of available diagnostic and prognostic strategies for the evaluation of stable angina patients: an observational assessment of the value of precatheterization ischemia. Economics of Noninvasive Diagnosis (END) Multicenter Study Group. J Am Coll Cardiol. 1999;33:661-669.

8. The American Board of Radiology. Maintaining the public trust in the era of healthcare reform: annual report 2010-2011. Available at: http://www.theabr. org/forms/ABR\%20annual\%20report\%202010-11.pdf. Accessed November 4, 2011.

9. Guiberteau MJ, Graham MM. ACR-SNM Task Force on Nuclear Medicine Training: report of the task force. J Nucl Med. 2011;52:998-1002.

10. Guiberteau MJ, Graham MM. ACR-SNM Task Force on Nuclear Medicine Training: report of the task force. J Am Coll Radiol. 2011;8:388-392.

11. The American Board of Nuclear Medicine: Certification. Available at: http://www. abnm.org/index.cfm?PageID=5044\&RPID=4999. Accessed November 15, 2011.

12. Accreditation Council for Graduate Medical Education: Number of Accredited Programs for the current academic year. Available at: http://www.acgme.org/ adspublic/reports/accredited_programs.asp. Accessed November 4, 2011.

13. Delbeke D, Graham M, Royal H, et al. Conjoint statement of the SNM, ACNM, and ABNM on credentialing and delineation of privileges for therapeutic procedures using radiopharmaceuticals. J Nucl Med. 2011;52:323-326.

14. Accreditation Council for Graduate Medical Education. Nuclear medicine program requirements. Available at: http://www.acgme.org/acWebsite/RRC_200/ 200_prIndex.asp. Accessed November 4, 2011.

15. The American Board of Radiology. Initial certification: nuclear radiology. Available at: http://www.theabr.org/ic/ic_nuc_landing.html. Accessed November 4, 2011.

16. The American Board of Radiology. Initial certification: diagnostic radiology. Available at: http://www.theabr.org/ic/ic_dr_landing.html. Accessed November 4, 2011.

17. Nicholson BT, Cohen MA, Harvey JA, Gay SB. Creating a new curriculum to prepare for the 2013 ABR testing changes: an academic residency perspective. J Am Coll Radiol. 2010;7:229-232.

18. Certification Board of Nuclear Cardiology. Exam statistics: certification examination in nuclear cardiology history and statistics. Available at: http://www. cccvi.org/cbnc/content_152.cfm. Accessed November 4, 2011.

19. American College of Radiology Web site. Available at: http://www.acr.org/ MainMenuCategories/about_us.aspx. Accessed November 15, 2011.

20. Association for University Radiologists Web site. Available at: www.aur.org. Accessed November 16, 2011.

21. Society of Chairs of Academic Radiology Departments Web site. Available at: www.scardweb.org. Accessed November 16, 2011.

22. Bhargavan M, Sunshine JH. The growing size of radiology. J Am Coll Radiol. 2008;5:801-805

23. Society of Nuclear Medicine Web site. Available at: http://interactive.snm.org/ index.cfm?PageID=14. Accessed November 15, 2011.

24. Harolds JA, Novelline RA, Guiberteau MJ, Metter D, Oates ME. Jobs and new initiatives in nuclear medicine education. J Nucl Med. 2011;52:17N-23N. 